\title{
Practice Spotlight: Drug Safety Pharmacist
}

\author{
Sandra Knowles, BScPhm \\ Drug Safety Pharmacist \\ Drug Safety Clinic \\ Sunnybrook Health Sciences Centre \\ Toronto, Ontario
}

$\mathrm{T}$ he Drug Safety Clinic, located within Sunnybrook Health Sciences Centre, is a multidisciplinary outpatient clinic that provides comprehensive assessments for patients with suspected adverse drug reactions. The clinic is recognized as a world leader in the management and diagnosis of adverse drug reactions. Although the clinic has been operational since 1985, it was in 1992 that the hospital provided funding for a part-time pharmacist to be associated with the clinic. Since then, Sandra Knowles, the drug safety pharmacist, has been involved in providing clinical services and in the research and educational activities of the Drug Safety Clinic.

About 300 patients visit the clinic each month. Upon initial referral, a patient will have a consultation with 1 of 4 physicians (a specialist in clinical pharmacology, dermatology, allergy/immunology, or internal medicine). The physician takes a thorough history in relation to the putative adverse drug reaction. Patient presentations encompass the entire spectrum of adverse drug reactions, although most patients have a history of an allergic drug reaction (e.g., urticaria, anaphylaxis, maculopapular drug eruption) or an idiosyncratic reaction (e.g., hepatotoxicity, toxic epidermal necrolysis).

For patients with complex medical histories involving many medications, Ms Knowles often participates in the initial interview. As well, for unusual and/or serious reactions, she conducts a thorough literature review to aid in assessing causality for the adverse drug reaction. Each of these detailed written reports is also sent to the referring physician. These reviews are integral components in the management of the patient, as previously published case reports provide insight into the onset of the reaction, associated symptoms, any testing that can be done, and, importantly, which drugs can be safely administered and which drugs must be avoided in the future.

Ms Knowles works closely with other members of the team to develop patient-specific therapeutic plans. The plans may incorporate diagnostic tests (e.g., skin tests, patch tests, oral challenges), desensitization procedures (e.g., with acetylsalicylic acid or clopidogrel), or various placebo trials (e.g., an $n$-of- 1 trial, in which 2 doses of placebo and 2 doses of active drug are administered to the patient in random order over a 4-week period). The infrastructure of the clinic includes the pharmacy manufacturing group, which prepares all testing and desensitization materials for the clinic. About $80 \%$ of all patients referred to the clinic undergo some type of testing. Ms Knowles undertakes follow-up for selected patients, and she also provides ongoing support to the patient during long desensitization periods (e.g., 1 month for allopurinol).

As mentioned above, Ms Knowles is involved in the research activities of the Drug Safety Clinic. This work includes developing new testing materials (e.g., abacavir patch tests, fluoroquinolone skin tests), identifying the genetic determinants of adverse drug reactions, and performing epidemiological research (e.g., role of gender in adverse drug reactions). Ms Knowles collaborates with researchers at both the national and the international level. She has published more than 90 peer-reviewed articles, as well as 15 book chapters, many pertaining to dermatologic adverse drug reactions.

Education is a significant component of the role of the drug safety pharmacist. Ms Knowles provides numerous in-house presentations to medical and pharmacy staff at the hospital, as well as presenting information about adverse drug reactions to local, national, and international audiences. She is a lecturer in the Leslie Dan Faculty of Pharmacy of the University of Toronto. In addition, the Drug Safety Clinic serves as a rotation site for Sunnybrook's ambulatory care resident and for students enrolled in PharmD programs across Canada.

Ms Knowles is also the manager of the Drug Safety Clinic and is responsible for human resources, budgetary issues, and other administrative functions. In addition to her involvement with the various outpatient activities related to the clinic, Ms Knowles also works closely with the hospital's division of Clinical Pharmacology in assessing patients who experience adverse drug reactions during their admissions. In her role as the drug safety pharmacist, Ms Knowles is an active member of the Pharmacy and Therapeutics Committee.

The position of drug safety pharmacist represents one of several opportunities for pharmacists to develop specialized roles in outpatient clinics in Canada. Other settings in which such specialized roles could be defined include the areas of dermatology (e.g., psoriasis, immunobullous diseases), family practice, and rheumatology. 